\title{
Reflections on the Is: Physics Penetrates Ontology
}

\author{
J.A. JANIK \\ H. Niewodniczański Institute of Nuclear Physics \\ Polish Academy of Sciences \\ Radzikowskiego 152, 31-342 Kraków, Poland \\ Dedicated to the memory of Professor Jerzy Prochorow
}

When considering questions such as What it means that something is? when? where? and how? we may for some purposes say that we are concentrating entirely on is - sometime, somewhere, somehow. This illustrates a transition from physical abstraction to ontological abstraction. However, we must remember - about Something - to say not only that it is, but also what it is. This is an old statement which says in a way that a being is "composed" of is and essence - esse et essentia. In this essay, we contemplate a connection between ontological abstraction and esse taken as a "component" of esse + essentia and show how physics relates to such considerations.

It seems to me that what is being sought for in questions such as when? where? and how? is connected to essence. Physics arrives at this via the equations of quantum mechanics, in particular via the Schrödinger equation. The results of this theory are only probable, since the wave function provides probability and not fact. (We do not know when a radioactive nucleus will decay, neither do we know where on a screen the trace of particle will show up, nor what its momentum is if we know its location - we generally only have probable answers to these questions.)

But we obtain all these answers, whether probable or certain, within the framework of an assumed abstraction. The concept of a material point may serve as an example of a physical abstraction. The interpretation of quantum mechanics also belongs to the class of physical abstractions (which perhaps may be awaiting improvement). Among these abstractions we find the concept of the reduction of the wave packet, which provides an actual is to an object. It thus provides a fact, not merely a probability - although this fact lies outside the scope of the quantum mechanical equations themselves (The reduction of the wave packet does not follow from the Schrdinger equation.) [1, 2]. 
This actual "real" is seems to be close to the is which occupies the first place in the "composition" esse + essentia, and which was thoroughly discussed by Thomas Aquinas and Martin Heidegger [3]. But in order to grasp this is by itself we must remove all traces of what it is by eliminating the content of the questions concerning when, where, and how. Hence we must abstract from everything except $i s$. In this way we arrive at the ontological abstraction.

I think that a method for achieving this degree of abstraction could be the integration of the probabilities over time, space, velocities, and other physical parameters. Having done this, we obtain "hard" answers to the questions when, where, and how; viz., sometime, somewhere, and somehow. Such answers may not be satisfactory as useful information, but they isolate is (esse). We do not know when the radioactive nucleus will decay, but the decay will be a fact at some time if we consider time as ranging from zero to infinity. This process is analogous to the process of marginalization in the theory of probability* [4]. Given, for example, two variables $x$ and $y$ of which only one, $x$, is of interest and an informational reservoir $I$, we can obtain the probability of $x$ when $y$ is marginalized as

$$
\operatorname{probability}(x \mid I)=\int \operatorname{probability}(x, y \mid I) \mathrm{d} y .
$$

I must admit here that the derivation of this formula treats $x$ and $y$ as propositions - statements that may or may not be true. Hence I do not know if the formula is valid when $x$ and $y$ are time and space coordinates and so on.

We made find it useful, for instance, when reflecting on the causality problem - when trying to find if a cause is, without considering when, where, or how.

However, we must be careful with the meaning of $i s$. We use this word in relation to a stone as well as to God or to electrons, but we must remember that there are linguistic as well as analogical differences in our uses of $i s$. If I say "the stone is" I am operating in the framework of colloquial language, and if I say "God is" I use is in an analogical sense. Even if I say "the electron is" I am also operating within an analogy. If I speak about the electron "flying" to the detector, I am not speaking about a fact but about the probability wave. The is of the probability wave has a different sense than an actual is. And if I speak about the local registration of the electron (via a reduction of the wave packet) I still do not quite speak colloquially, because I must remember that the momentum coordinates still remain involved in the superposition of states.

When considering borderline matters between philosophy and physics, we cannot omit time. Thomas Aquinas introduces time by pointing out that what is undergoes changes. This permits conscious observers to distinguish between "before" and "after" when considering change. Thomas uses the word "numbering", meaning numbering before and after. He writes, "Time is applicable to what is material. It is based on motion, on what is before and what is after. But its

${ }^{*}$ I obtained this information from Professor Ludwik Dobrzyński. 
deepest basis is in the act of a numbering soul". Thomas uses the word anima, which I interpret as a conscious observer [5].

In our colloquial language we distinguish between now, past, and future. When we say $i s$, we immediately notice no duration; this is characteristic of now. What is now shows up and immediately disappears, replaced by another now. Edith Stein writes, "My is I find on a knife's edge. What we feel as a duration is a continuous transfer of the touching point. This is an existential duration, which creates time as its space. To be presents itself as to become" [6].

A quantum physicist believes that his theory provides him information about the states of an object by giving him the probabilities of their occurrence. Every such occurrence happens through the reduction of the wave packet. These reductions happen in a flash that has no duration, just as it is with the now of Edith Stein. Here is is reached in an almost colloquial sense, although limited to a particular property; e.g., place, which was actualized through the reduction of the wave packet. This $i s$ has no duration. But the next reductions of the wave packet provide more of such instantaneous flashes. If we have the right to ascribe these to the same object, we obtain in this way a sequence of "befores" and "afters". We thus obtain time from the point of view of the physicists.

It seems to me that ideas concerning time provided by Thomas Aquinas and Edith Stein on the one hand and physicists on the other are in agreement. If the reduction of the wave packet demands a conscious observer, as many physicists believe but many deny, we have an agreement even more pronounced, since, according to the Thomas Aquinas, an anima is necessary for the perception of the time.

Thus we are led to the question of whether or not time is simply a series of timeless flashes.

\section{Acknowledgments}

I am grateful for suggesting my contribution to the issue of Acta Physica Polonica A in memoriam to Professor Jerzy Prochorow.

My thanks are also due to Professor Scott Desjardins from the University of Rhode Island for stimulating discussions and help in editing work of this essay.

\section{References}

[1] J.A. Janik, Concepts of Physics 3, 137 (2006).

[2] G. Greenstein, A.G. Zajonc, The Quantum Challenge, Jones and Barlet Publ., Boston 1997.

[3] J.D. Caputo, Heidegger and Aquinas, Fordham University Press, New York 1982.

[4] D.S. Sivia, W.I.F. David, K.S. Knight, S.F. Gull, Physica D 66, 234 (1993).

[5] Thomas Aquinas, Super Physicorum IV, 17.

[6] Edith Stein, Endliches und ewiges Sein, Herder, Freiburg 1986, p. 60. 